\title{
La caricature sociale dans les albums de Jean Bruller : une Comédie humaine de l'entre-deux-guerres
}

Avant de devenir l'un des écrivains de la Résistance les plus lus grâce à sa célèbre nouvelle Le silence de la mer (1942), Jean Bruller, connu sous le pseudonyme littéraire de Vercors, a longtemps exercé le métier de dessinateur. Écriture et dessin se côtoient pourtant dès les premières publications de l'artiste, avant de laisser une place presque exclusive à la création littéraire avec l'avènement de la Seconde Guerre mondiale. Consacrés en grande partie à la satire et à la critique de la société française de l'entredeux-guerres et caractérisés par un humour particulièrement mordant et ironique, ces albums proposent un système hybride de texte et d'image propice à la réalisation de la caricature.

Ainsi, La danse des vivants (1932-1938) aspire à illustrer la comédie humaine du XX ${ }^{e}$ siècle, mettant en place une vision générale d'une certaine hauteur intellectuelle. Miroir critique de l'homme contemporain, la caricature de mœurs (Tillier, 2015, p. 32) fait ressortir l'universalité de certaines caractéristiques humaines tout en se servant d'un fin humour railleur. La prétention de Jean Bruller de construire une vision plutôt globalisante et détachée faiblit pourtant, progressivement, avec la présence prégnante des menaces de guerre. Visions intimes et rassurantes de la guerre (1936), dans un tout autre registre de la caricature sociale du dessinateur, propose une focalisation sur ce contexte précis par des personnages égoïstes et intéressés par les «bienfaits» d'un possible conflit. La caricature de type, visant un large creuset de catégories sociales (Tillier, 2015, p. 33), vient s'ajouter à l'appareil critique mis

- María de los Ángeles Hernández Gómez - docteure en littérature française, ATER à l’Université Clermont Auvergne. Adresse de correspondance : Centre de Recherches sur les Littératures et la Sociopoétique, 4 rue Ledru, 63057, Clermont-Ferrand, France ; e-mail : mangeleshernandez21@gmail.com

ORCID iD : https://orcid.org/0000-0001-5883-5420 
en œuvre par le dessinateur, image aussi de l'effritement du pacifisme d'avant-guerre de l'artiste.

Imprégnés à la fois d'un désir de permanence et d'un investissement inévitable dans le présent, ces deux albums donnent vie à un mécanisme de caricature sociale très personnel qui échapperait à la perte de son acuité et au vieillissement propre au passage du temps (Roy, 1974, p. 23). Il s'agira de montrer comment l'articulation des instances visuelle et textuelle est mise au profit du projet brullerien et d'analyser quel est le dialogue spécifique qui s’établit entre l'œuvre, la pensée de l'artiste et le contexte historico-social de l'époque par le biais de la caricature.

\section{Caricaturer I'homme du XX' siècle}

La danse des vivants suppose l'ouverture de l'art de Jean Bruller à l'homme social et une prise de distance définitive avec ses premières publications, où l'ombre du dessinateur à la recherche de son identité empêchait la réflexion d'atteindre une portée globale. Échappant à l'emprise autobiographique, le projet ne sera pourtant pas détaché de l'individu mondain, véritable représentant de la comédie humaine brullerienne. Brusquement arrêté avec l'approche de la Seconde Guerre mondiale pour n'être jamais repris, cet album constitue l'entreprise la plus ambitieuse de Jean Bruller comme dessinateur. Il s'agit d'un ouvrage réunissant 160 estampes, publiées sous forme de Relevés Trimestriels entre 1932 et 1938. Apparaissant de manière aléatoire et désordonnée dans chaque numéro, les estampes s'organisent pourtant à partir d'une cohérence significative de base : «le sujet pourrait s'énoncer, si j’étais un auteur grave : Vie de l'Homme d'Aujourd'hui » (Bruller, 2000, p. 373).

En dehors de l'influence balzacienne de l'œuvre, un autre exemple littéraire viendra accélérer le mûrissement de cette production : la publication en avril 1932 des deux premiers volumes du roman fleuve Les hommes de bonne volonté de son ami et écrivain Jules Romains. Les correspondances avec ce dernier, que Vercors reproduit en partie dans Les occasions perdues (1982), confirment le goût pour l'«imposture comique», la communion "dans une égale haine de la guerre, un égal amour de la paix » (p. 23) et les intérêts intellectuels et idéologiques qu'ils ont en commun. Ces quelques échanges entre les auteurs délimitent à la perfection la dynamique des créations brulleriennes dans ces années trente : intrinsèquement comiques, malgré le pessimisme qui baigne l'œuvre de l'artiste à l'époque, et en lien indéniable avec la société et l'actualité de son temps : «Je désire que mes dessins représentent les notes d'un témoin, et non les constructions d'un esprit; il me faut recevoir mes idées du dehors, et non les faire jaillir de l'intérieur» (Bruller, 2000, p. 376).

Refusant «l'art pour l'art», Jean Bruller dessine pour dire, pour transmettre et faire réfléchir, d'ailleurs, une fois que le dessin se révélera insuffisant, il changera de moyen d'expression pour l'écriture. De fait, après la livraison unique que le dessinateur fait en 1935 de La danse des vivants, la publication s'arrête pour n'être reprise qu'en 1938. 
L'auteur prend soin d'expliquer les raisons de ces trois ans de silence : les événements politiques et sociaux des dernières années l'auraient empêché de dessiner pour une publication qui visait des idées générales et en quelque sorte intemporelles, car en lien direct avec la condition humaine. C'est pendant cette pause qu'il crée et publie Visions intimes et rassurantes de la guerre (1936). Cependant, existe-t-il un véritable écart entre les deux travaux ? Plus qu'une rupture, nous interprétons l'album de 1936 comme une focalisation, comme une mise en exemple concrète des travers que Jean Bruller se plaît à dénoncer dans ses relevés et qui trouvent dans le conflit imminent un terrain propice d'exploitation. En fait, l'homme de La danse des vivants, n'est-il pas celui du $\mathrm{XX}^{e}$ siècle, marqué d'une manière ou d'une autre par la menace, la présence ou le souvenir de la guerre?

Malgré des perspectives différentes, les deux albums partagent un ton humoristique et satirique, derrière lequel se devine une certaine amertume et dont la vocation dénonciatrice donne forme à la caricature sociale brullerienne. Il ne s'agit point ici d'une caricature amplificatrice qui met en valeur les traits accentués des personnages. Si les dessins exposent à maintes reprises des expressions marquées ou exagérées, c'est dans l'objectif d'accentuer l'attitude et les sentiments des personnages : le ridicule est moins dans leur physique que dans leurs actions, leurs pensées, leurs rêves, leurs prétentions inutiles. Dès lors que le dessinateur cherche une certaine hauteur de vue sur la société qu'il représente, les protagonistes ne sont plus la transposition de personnalités quelconques qui personnifieraient les événements et les faits politiques (Tillier, 2005, p. 21). L'artiste situe son œuvre loin du portrait-charge, du fait même qu'il ne vise pas des hommes concrets, mais l'homme ${ }^{1}$ comme entité. La caricature brullerienne se bâtit sur une mise en scène d'ensemble, sur la subtilité du crayon qui aspire à montrer le moindre repli de la psychologie des personnages, mais qui prend soin par la même occasion des détails de la composition, profondément significatifs dans la dénonciation envisagée.

Le texte joue aussi un rôle fondamental dans le système caricatural brullerien, ce qui ne fait que confirmer la place protagoniste de l'écriture dans l'ouvre de l'auteur. Nous aurions du mal à définir certaines de ses productions comme des «albums commentés » ou même comme des "textes imagés», tant écriture et dessin se complètent dans des créations iconotextuelles conçues comme de véritables totalités indissolubles (Nerlich, 1990, p. 268). Cette interdépendance du trait et du verbe (Tillier, 2005, p. 204-205) s'avère d'ailleurs la clé indispensable pour comprendre la pluralité de sens des publications. Dans le cas de La danse des vivants, les légendes qui accompagnent chacune des illustrations s'articulent sur différentes fonctions du langage (le non-sens, l'absurde, le calembour, le proverbe, l'ironie, etc.), qui déterminent le ton du dialogue avec les dessins et créent ainsi les effets humoristiques recherchés

1. Nous tenons ici à écarter la majuscule du nom «homme» du fait que Jean Bruller, bien qu'aspirant à un regard universel, reste tout de même circonscrit à la société occidentale, plus spécifiquement française, du XXe siècle. 
(critique, dénonciation, ridiculisation, etc.). Dans Visions, la présence du verbe se fait plus imposante, car le texte assume la narration et l'interprétation des illustrations ; nous verrons comment la disposition spatiale de la composition et le développement complémentaire des deux instances donnent lieu à une satire de plus en plus acide.

\section{Les Danses macabres de Jean Bruller}

La formule choisie par Jean Bruller pour intituler son grand projet invite le lecteur à faire des rapprochements avec les Danses macabres du Moyen-Âge, actualisées et renouvelées ici par la pensée et la réalité modernes. L'artiste ne reprend pas le thème allégorique du pouvoir égalisateur de la mort ; en revanche, l'ouvrage suit la même dynamique de dénonciation des travers et des vices humains. Le pessimisme et l'absurde complètent l'œuvre donnant lieu à un humour noir proche des compositions moyenâgeuses évoquées.

La danse des vivants doit se comprendre avant tout comme l'expression artistique de la crise existentielle que vit l'auteur dans ces années trente : «L'Humanité [...] servirait à quoi ? À Rien, ni moi non plus. Le résultat est le même. Je suis, nous sommes tous là, mes frères, sans raison d’être " (Vercors, 1991, p. 62-63). Cependant, loin de se complaire dans le nihilisme, Jean Bruller décide de dénoncer les efforts stériles de l'homme pour surmonter l'absurdité de son existence, d'ironiser et de mettre en avant le ridicule qui frappe toutes les illusions, les travers, les vanités, les ambitions ou les rivalités des individus. C'est par la combinaison de cet humour satirique et de pessimisme que le dessinateur donne vie à la caricature de mœurs de sa comédie humaine du XXe siècle, et ce principalement dans le deuxième tome de l'ouvrage, intitulé «Copies conformes». L'ironie est, dans ces dessins-ci, plus puissante que jamais, permettant de souligner davantage le caractère dérisoire de l'homme et d'incorporer une voix dénonciatrice de la vanité de certaines de nos actions.

Nous y trouverons, par exemple, un grand nombre d'illustrations consacrées à la réflexion sur la liberté de l'homme et sur la conscience qu'il en a. Se croyant complètement maître de ses décisions, ses désirs, ses besoins ou ses achats, il vivrait dans l'ignorance des divers facteurs qui conditionnent son existence et qui finissent par l'aliéner (Clavel, 1970, p. 47). Jean Bruller met en scène ces contraintes invisibles, interprétées comme des choix personnels : la tranquille promenade en famille du dimanche devient une pénible lutte pour prendre sa place au milieu de la foule. La masse continue ainsi de suivre une tradition, malgré l'évidente disparition du plaisir de l'activité, devenue presque une corvée. C'est de même l'opportunité de représenter l'aspect impersonnel, presque déshumanisant, de ces activités sociales; le dessin en rend compte par des personnages de dos qui, les uns après les autres, avancent dans une direction commune en guise de manifestation dérisoire. Le titre, présenté sous forme de légende et situé en marge de l'image, en regard de laquelle il fonctionne, opère le tournant caricatural sur l’ensemble, procédé qui se répétera tout au long de l'album. 


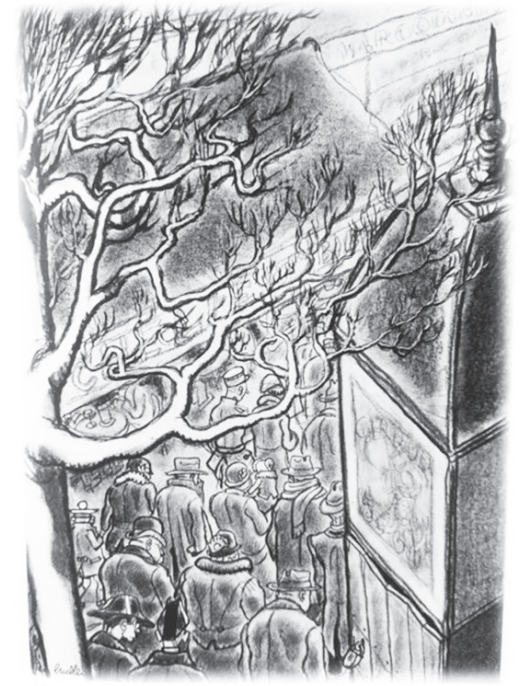

Figure 1.

Dimanche!

Dimanche!

À cette occasion, l'écart entre l'enthousiasme textuel de "Dimanche! Dimanche ! » (fig. 1) et l'image angoissante de la masse dans les rues permet au caricaturiste de disséquer et de railler en même temps les mœurs, non sans un arrière-goût morose.

Plus loin, l'intitulé descriptif «Fin de journée ou la vie oisive» (fig. 2) accompagne l'illustration d'une femme épuisée sur son canapé après une longue journée de shopping qui ne semble pas lui avoir apporté la détente ou la joie recherchées, bien au contraire. Seule, dans un appartement qui présente toutes les commodités d'une situation économique aisée, l'expression de la femme laisse entendre la banalité de la consommation. La société de consommation est à plusieurs reprises critiquée par des illustrations qui restent aujourd'hui plus actuelles que jamais. Dans «Capitulation
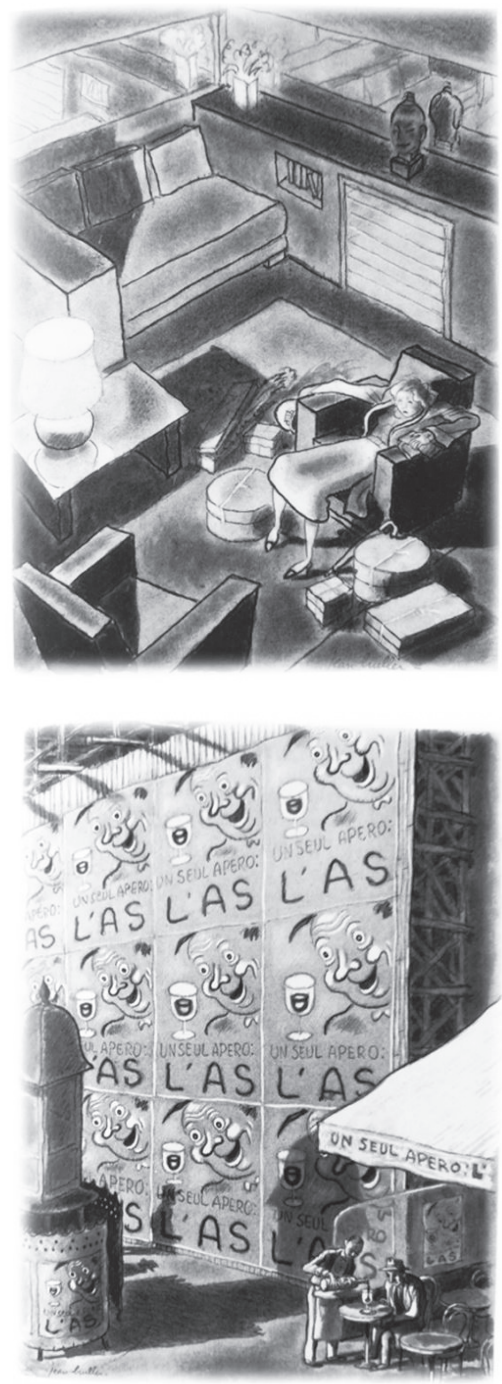

Figure 3. Capitulation ou le libre arbitre ou le libre arbitre» (fig. 3), le dessinateur met en image la forte influence de la publicité dans nos choix, qui se voient inconsciemment réduits et guidés. Visant la standardisation et l'unification des goûts des clients, elle finit par les soumettre, d'abord par un sentiment de nécessité, puis par l'achat de certains produits au détriment d'autres. L'illustration se sert du jeu des tailles pour mettre en scène l'homme qui, petit et déterminé, se voue à suivre les choix qu'on lui impose, cette fois, depuis la mode publicitaire. Conciliant l'imitation et la déformation de la réalité (Vaillant, 2014, p. 259), les affiches géantes occupent presque la totalité de l'espace pictural; le dessinateur 


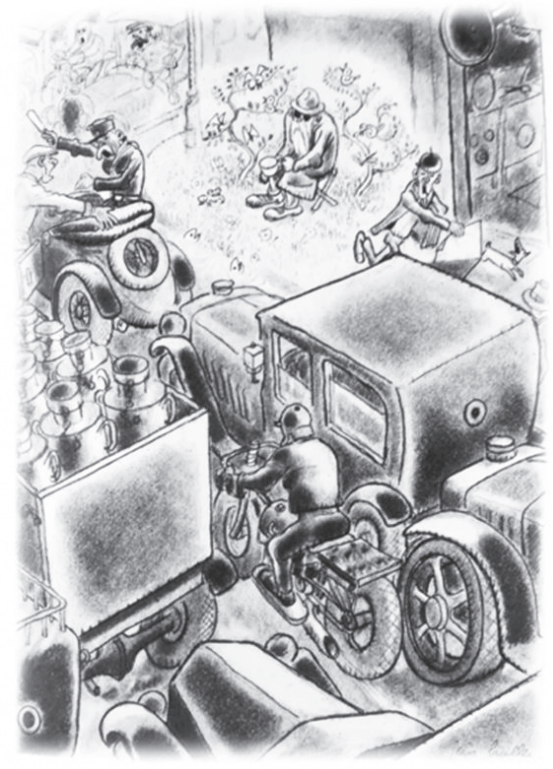

Figure 4. Symphonie pastorale ou le bienheureux aveugle et sourd

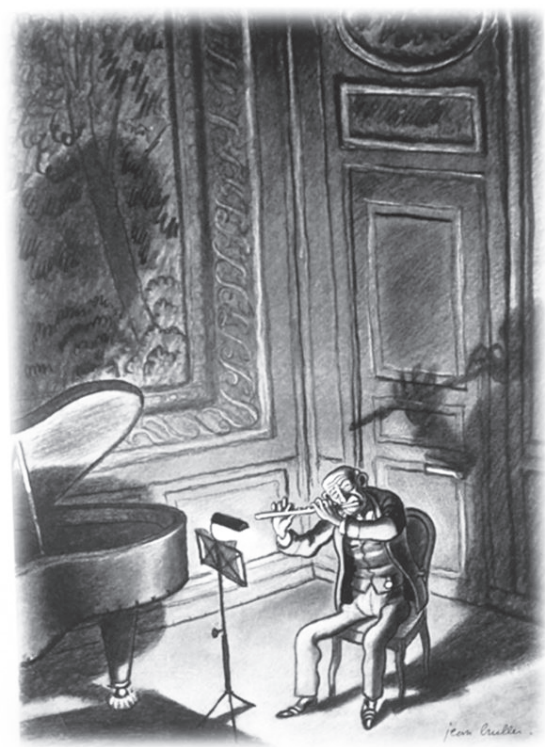

Figure 5. Le Marchand de canons reprend dans son ouvrage l'une des formules typiques du système publicitaire, à savoir la répétition constante des slogans et des images, et critique en passant les lobbies et le monopole de certaines marques ou entreprises. D'un point de vue textuel, la comparaison de notions aussi opposées que " capitulation » et « libre arbitre » finit d'affirmer l'impuissance de l'individu moderne face au système oppressant de consommation.

La caricature brullerienne s'attaque aussi à la course effrénée de l'homme du $\mathrm{XX}^{\mathrm{e}}$ siècle pour le progrès, qui semble en toute évidence avoir négligé les besoins réels de la société. Le numéro 12 des Relevés Trimestriels (novembre 1934) consacre quelques images à cette nécessité de progresser, devenue éternelle parce qu'inefficace. "Symphonie pastorale ou le bienheureux aveugle et sourd» (fig. 4) montre la joie des avancées dans le domaine des transports qui rythment une vie quotidienne, désormais marquée par les embouteillages, le bruit assourdissant des voitures et le stress continuel dans les rues. Pour sa part, "Cafard» évoque la face cachée du progrès, la complète solitude de l'homme dans un océan de bienfaits matériels. L'échafaudage critique mis en place par Jean Bruller dépasse par des exemples comme ceux-ci les dessins indépendants pour établir de même un dialogue entre les différentes illustrations qui constituent l'album.

Cependant, les conditions de l'existence humaine, constamment raillées et dénoncées dans La danse des vivants, semblent ne pas être un fardeau définitif pour l'homme moderne ; elles ne le conditionneraient jamais complètement (Arendt, 1961, p. 20). Cette «échappatoire» possible que Jean Bruller offre dans le dernier chapitre de son album constitue la plus haute expression de l'ironie de la publication. Retenons les trois premières illustrations du chapitre : «Le Marchand de canons» (fig. 5), «Le Mouchard» (fig. 6) et «Le Multimilliardaire» (fig. 7). Les titres se heurtent à des représentations qui restent 
très éloignées, voire opposées, à l'idée que l'on pourrait se faire des personnages concernés ; la dissociation du texte et de l'image atteint ici son paroxysme. Ainsi, celui qui s'enrichit avec la guerre fait preuve de ses délicats talents musicaux, alors que le sycophante et le multimilliardaire profitent de leur complicité avec la nature en toute simplicité. Le contraste est tel que le lecteur, interpelé à coopérer, reste sur ses gardes, se méfiant de la véritable personnalité des protagonistes. Dans son édition critique de La danse des vivants, Alain Riffaud indique qu'il s'agirait moins d'inciter à un rachat des vices ou des travers de l'homme que de souligner la possible existence d'une vertu (Bruller, 2000, p. 386). Par cette ouverture, tout comme par le ton caricatural et satirique qui structure l'album, Jean Bruller se refuse à s'enfermer dans une vision pessimiste irréconciliable mais laisse, au contraire, la porte ouverte à la foi en lêtre humain par l'humour. Un indice sans doute de l'engagement humaniste que l'artiste entreprendra dès le début de la Seconde Guerre mondiale.

\section{Caricature et guerre, une question de points de vue}

Cependant, cette vision d'espoir en l'homme reste très floue jusquà ce que Jean Bruller change définitivement de forme d'expression en 1942 par son entrée dans la résistance intellectuelle. L'approche de la guerre nourrit, au contraire, la méfiance de l'artiste vis-à-vis de l'attitude de toute une partie de la société eu-

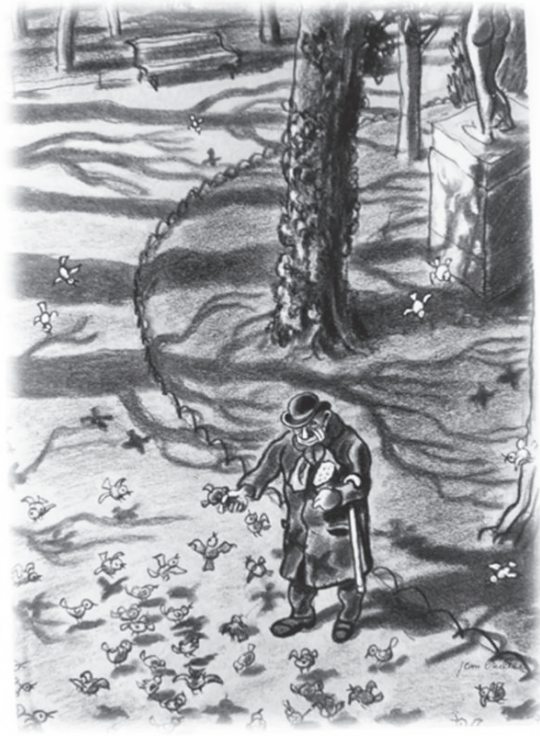

Figure 6. Le Mouchard

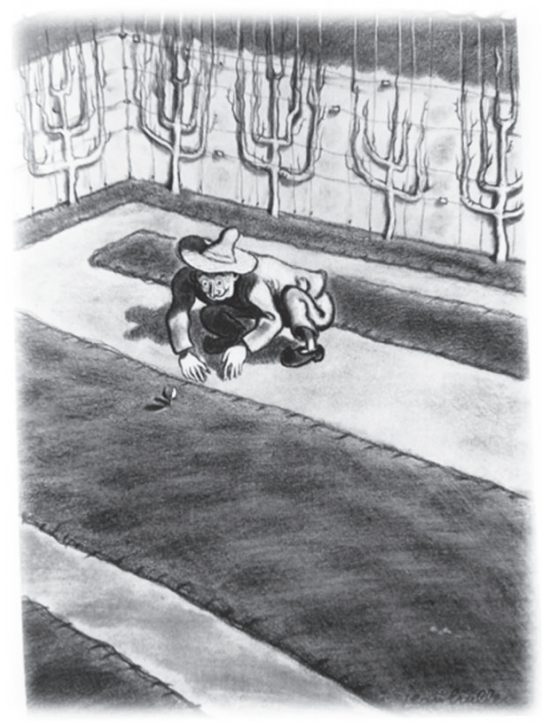

Figure 7. Le Multimilliardaire ropéenne, méfiance qui donnera lieu à la création de son album Visions intimes et rassurantes de la guerre, qui voit le jour en 1936. Cet ouvrage propose un éclairage tout à fait différent de celui de La danse des vivants, par une représentation de l'homme qui abandonne des prétentions universelles en faveur d'un cadre beaucoup plus concret et reconnaissable, ce qui renforce la contondante critique de l'album. 
Par la publication de Visions, Jean Bruller s'inscrit dans le sillage d'un grand nombre de productions qui, en cette moitié des années trente, font du thème de la paix, et en conséquence de la guerre, un véritable leitmotiv artistique ${ }^{2}$. Les correspondances de l'auteur rendent compte de l'omniprésence du conflit, devenu presque une hantise. Roger Martin du Gard, dans ses échanges réguliers avec Jean Bruller, parle de son Été 1914 (1936) comme d'une «longue préface» à son album, tellement les estampes du dessinateur semblent, à ses yeux, rendre compte des "hésitations de l'esprit", "de la sévérité», «de l'ironie», "de l'indulgence», «de la pitié», «de tout cela superposé et jaillissant» (Martin du Gard, 1936), propres à l'époque. En cette année 1936, Jean Bruller ne peut plus ignorer des réalités contemporaines, telles que la guerre civile qui vient d'éclater en Espagne ou encore la montée fulgurante de l'extrême droite européenne ; le tout dans un contexte d'insouciance généralisée. Ce basculement vers l'actualité de son temps confronte le dessinateur à ses convictions pacifistes par une remise en question qui se poursuivra jusqu'à l'Occupation, mais qui n'est pas encore mûrie au moment de la publication de Visions :

- [...] «Pacifiste à tous crins», disiez-vous. Quand avez-vous cessé de lêtre?

- Très tard. Très tard. Je n'étais pas aveugle mais je m’obstinais à penser : Tant qu'il n'y a pas la guerre tout peut encore survenir, un attentat contre Hitler, une rébellion de ses généraux... Même en 1936, lors du coup de poker de Hitler (pas encore prêt) envahissant la Rhénanie, je souhaitais encore le compromis. Je n’en suis pas fier. (Vercors, 1991, p. 75)

Si les positionnements d'une majorité des intellectuels se produisent après les accords de Munich, comme ce fut le cas de Jean Bruller, le mouvement pacifiste de gauche se réduit progressivement au fur et à mesure que l'agressivité extérieure du nazisme oblige à prioriser la lutte antifasciste sur la défense de la paix (Olivera et Offenstadt, 1993, p. 53). C'est dans cette dynamique de prise de conscience que le dessinateur arrive à penser la guerre comme quelque chose, sinon d'inévitable, du moins de très probable.

La comédie humaine brullerienne garde ici une certaine empreinte de pessimisme, né essentiellement de l'incrédulité du peuple face à des changements qu'il croit « inoffensifs ». Cependant, Jean Bruller n'hésite pas à réinvestir le ton satirique déjà typique de son activité créatrice pour explorer ce qu'il y a de plus égoïste et intéressé en l'homme dans un contexte pareil : «Les planches de l'album représentaient [...] comment une variété de personnages types imaginaient la guerre prochaine sous des couleurs riantes, espérant y trouver leur profit» (Vercors, 2002, p. 811). Visions ferait donc partie de la «préparation» de l'artiste au conflit par la critique acerbe envers ceux qui y voyaient, au-delà de la catastrophe générale, un moyen d'enrichissement

2. Marchands de canons de Maurice Rostand (1933), Le journal d'un homme de quarante ans de Jean Guéhenno (1934), La guerre de Troie n’aura pas lieu de Jean Giraudoux (1935) ou La nausée de JeanPaul Sartre (1938). 
et de réussite. Jean Bruller organise par cette occasion sa caricature sociale autour du type satirique, défini par Bertrand Tillier comme un personnage " dont on considère qu'il réunit un ensemble de caractères, qualités et défauts, physiques et moraux, traduisant l'essence d'un groupe donné d'individus [...], détaché de la société pour être mieux observé, moqué ou dénoncé» (Tillier, 2005, p. 29).

Ainsi, dans "Vision intime de la guerre d'Armand Lorraine de l'Académie française", l'académicien regrette les récits de guerre qui lui ont donné son siège à la prestigieuse institution, mais qui sont tombés dans l'oubli, démodés par une guerre déjà lointaine. Il rêve de la reprise des combats pour mettre en page ses héros et la défense de la patrie française. De son côté, l'industriel Robert Lefrançois gère ses comptes pour faire progresser son industrie d'artillerie (fig. 8). Aucun domaine ne semble lui convenir à exception de la guerre, qui multiplierait ses revenus sans être obligé de moderniser ses usines et qui, de plus, lui permettrait d'échapper à la mobilisation, étant donné qu'il contribuerait à l'effort de guerre par son travail. Suivant cette dynamique, Jean Bruller rapporte les arguments d'un médecin, d'un député, d'un chef de gare et même de la gérante d'un magasin de dentelles. Des personnages appartenant à des domaines complètement différents y sont réunis sur la base d'une semblable mesquinerie. Cependant, force est de constater que l'album s'attaque avec une spéciale virulence à la haute bourgeoisie, aux représentants de la classe politique et aux membres de l'armée.

En dehors de la présentation de ce large creuset de la société française par des types satiriques, ce nouvel album de Jean Bruller est particulièrement intéressant par son organisation matérielle, construite sur un jeu de points de vue déjà classique dans d'autres publications de l'artiste, telle Un homme coupé en tranches (1929). Certains pourraient le définir par sa nature hybride comme un "album illustré » : les images présentent une prépondérance, mais le texte est constamment mis en valeur par la place qu'il occupe dans la distribution de l'espace et par la narration qu'il prend en charge (Van der Linden, 2008, p. 52). La mise en page de chacune des caricatures proposées se fait sur un patron unique maintes fois répété ; il en résulte des représentations indépendantes dont la lecture peut à tout moment être arrêtée, l'ordre changé, sans que cela nuise pour autant à la compréhension générale de l'ouvrage.

Chaque représentation se développe sur une double page : à gauche une brève présentation du personnage concentre et singularise l'attaque sur un individu quelconque en donnant son nom de famille, attaque élargie ensuite par l'évocation 


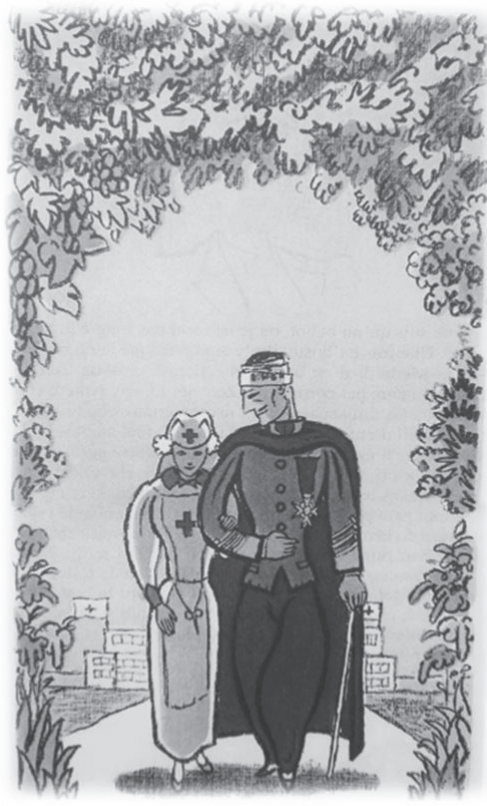

Figure 9. Vision intime de la guerre de Marceline Merlet, gérante des «Mille Dentelles»

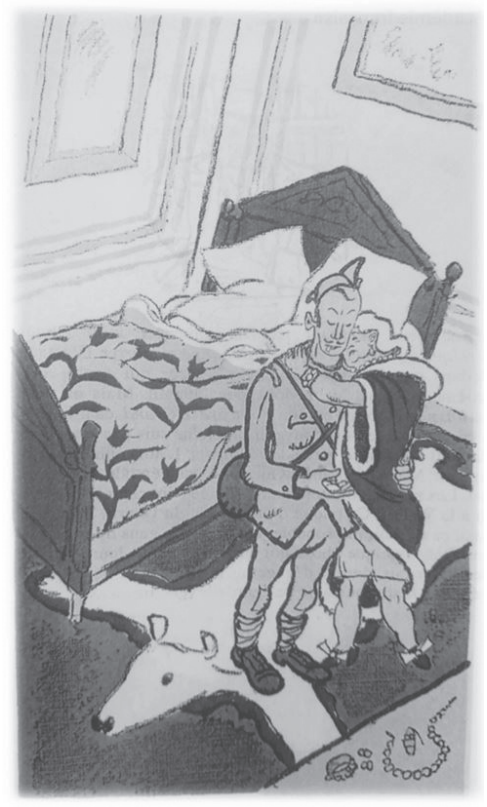

Figure 10. Vision intime de la guerre de Manuel Trécult, dit Manolo-Les-Belles-Feuilles du métier du protagoniste, ce qui en dit souvent beaucoup de la classe sociale à laquelle il appartient (journaliste, tenancier, secrétaire de l'ambassade, ex-étudiant en droit, maître de forges et pétrolier, etc.). Suivent le portrait dessiné de chacun des personnages, souvent représentés d'un air pensif ou grave, et un texte bref qui reproduit leurs pensées autour des situations de guerre désirées. En miroir et occupant toute la page droite, la mise en images des propos écrits.

La caricature sociale brullerienne prend forme dans ce dialogue texte-images, dont le positionnement en miroir multiplie les effets comiques. Par exemple, dans "Vision intime de la guerre de Marceline Merlet, gérante des "Mille Dentelles" » (fig. 9), l'artiste parodie les aspirations d'une jeune fille célibataire : sauver la vie d'un charmant capitaine blessé qu'elle épouserait par la suite. Le dessinateur s'amuse dans son texte à reproduire leur première conversation après le rétablissement du capitaine. Le lecteur ne pourra pas s'empêcher de sourire à la lecture d'un échange marqué par des expressions clichées comme «Je vous dois la vie ", "Vous êtes près de moi comme un ange delumière » ou encore «Taisez-vous, vous allez vous fatiguer»(Bruller, 2002, p. 60). L'auteur joue aussi avec le stéréotype de la jeune infirmière dévouée pour son travail et sa patrie, admiratrice du « héros de guerre ". En face, l'image de rêve du couple sortant de l'hôpital main dans la main matérialise une fin heureuse, d'autant plus dérisoire qu'elle contraste avec le visage frustré de la jeune fille qui, dans le portrait de gauche, attend sa chance dans un magasin vide de toute clientèle.

Dans «Vision intime de la guerre de Manuel Trécult, dit Manolo-Les-Belles-Feuilles » (fig. 10), la caricature se construit dès l'intitulé de l'illustration. La dynamique de présentation des personnages se voit altérée par le statut particulier du protagoniste : on ne lui connaît ni office ni travail. Le portrait d'un homme à casquette, chemise et veste, petite moustache bien soignée et cure- 
dent à la bouche, annonce presque naturellement le profil de ce voyou de province : son objectif est de partir à la guerre, de se faire blesser et, une fois à l'hôpital, d'essayer de charmer l'une des vieilles dames riches qui aident charitablement les établissements de santé en période de conflit armé. Il rend compte de son plan d'action, magistralement inspiré de l'expérience du Père-Saint-Médard : « il en avait collectionné plus de trente, à ce qu'il dit » (Bruller, 2002, p. 62). Jean Bruller peaufine au maximum le discours de Manuel Trécult par un langage très populaire, voire vulgaire, en complète adaptation aux objectifs hautement louables qu'il s'est donnés. En exemple, les expressions dont il se sert pour parler des femmes qu'il vise à attirer : "rombière d'âge encore tendre » ou encore "vache » à traire (p. 62). Le dessin d'une dame pendue à son cou et parée de nombreux bijoux, dans une chambre somptueuse, renvoie à la réalisation du rêve de Manuel, situation qui semble pourtant très inatteignable au vu du savoir-faire inexistant du protagoniste. Le clivage entre le point de départ de Manolo-Les-Belles-Feuilles et ses prétentions étant incommensurables, il ne peut qu'apparaitre ridicule aux yeux du lecteur.

Ces propositions plus originales cohabitent avec des images habituelles du monde de la caricature autour, par exemple, de la culture scatologique du bas corporel ( Vision intime de la guerre de Paul Brousset, chirurgien ») ou de la débauche sexuelle ( Vision intime de la guerre de Jules Chauvepie, ex-droguiste à Couilly-du-Loir »). Dans un autre registre, nous trouvons aussi des allusions spécifiquement politiques et patriotiques qui tournent au dérisoire l'" honneur " de mourir au nom de la patrie. En guise d'anecdote, signalons « Vision intime de la guerre du lieutenant-colonel Dupin officier breveté» (fig. 11), dont l'aspect humoristique semble aujourd'hui atténué par la grande précision historique que le dessin a eu par la suite : il annonce, à quelques jours près (le 12 mai 1940), l'invasion allemande et la destruction de la défense de Bapaume, qui aura lieu le 18 de ce même mois.

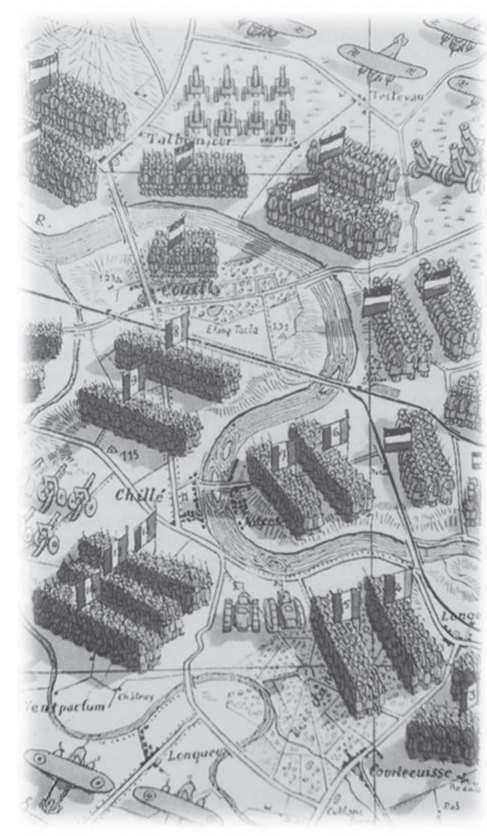

Figure 11. Vision intime de la guerre du lieutenant-colonel Dupin, officier breveté

\section{Conclusion}

Cette « vue de près » de la comédie humaine brullerienne annonce en quelque sorte le tournant dans l'œuvre artistique de l'auteur, qui pourra difficilement échapper à l'emprise que l'actualité sociale exerce sur son travail de création. Malgré la présence presque fantasmatique de la guerre, le dessinateur arrive encore à articuler 
sa proposition sur un ton humoristique, qui disparait progressivement au fil des publications jusqu'à l'éclatement de la guerre. L'humour reste d'ailleurs l'une des marques d'identité des travaux de Jean Bruller, qu'il marie à tour de rôle à la caricature, à la satire, à l'absurde et même au pessimisme pour mieux essayer de saisir la condition humaine par l'art: « Toujours je m'étais efforcé à travers l'humour et la satire, d'atteindre au permanent, aux problèmes éternels de l'homme face à sa condition sur terre " (Vercors, 2002, p. 810). La caricature se présente dans ce contexte comme un outil précieux pour exploiter les formes multiples de l'humour, visuelles ou écrites, en même temps qu'elle dénonce ou raille certaines attitudes sociales à la manière d'un pamphlet. Par une vision générale ou par une vision plus concrète, c'est ce ton caricatural qui permet de souligner les spécificités de la société moderne très révélatrices, en définitive, de la condition humaine. Vercors parlera d'ailleurs, dans son entretien avec Gilles Plazy, du rire comme l'un des modes d'expression par excellence de l'homme, quelque chose qui lui est propre et qui le définit (Vercors, 1991, p. 49). Cependant, il se dira incapable de continuer son projet sur ce ton humoristique ; la deuxième pause en 1938 de la série La danse des vivants, à cause des menaces de guerre, sera définitive : «La catastrophe avait tari en moi toute source d'humour et d'inspiration" (Vercors, 1991, p. 27)3. Il faudra en effet attendre la fin du conflit et le dépassement des questionnements éthico-philosophiques issus de la guerre pour voir resurgir dans la production vercorienne l'ironie et l'humour qui ont marqué les débuts de Jean Bruller dans le monde de l'art.

\section{RÉFÉRENCES}

Arendt, H. (1961). Condition de l'homme moderne. Paris : Calmann-Lévy.

Bruller, J. (2000). La danse des vivants. A. Riffaud (éd.). Le Mans : Création \& recherche.

Bruller, J. (2002). Visions intimes et rassurantes de la guerre. A. Riffaud (éd.). Paris : Omnibus.

Clavel, M. (1970). Qui est aliéné ? Critique et métaphysique sociale de l'Occident. Paris : Flammarion.

Martin du Gard, R. (1936). Lettre de Roger Martin du Gard à Jean Bruller. Paris : Fonds Vercors. Nerlich, M. (1990). Qu'est-ce qu'un iconotexte ? Réflexions sur le rapport texte-image photographique dans La Femme se découvre d'Évelyne Sinassamy. Dans A. Montandon (éd.). Iconotextes (p. 255-302). Clermont-Ferrand : Orphys.

Olivera, P. et Offenstadt, N. (1993). L’engagement pour la paix dans la France de l'entre-deuxguerres : un ou des pacifismes? Matériaux pour l'histoire de notre temps, vol. 30 / 1, 53-56.

3. Le travail n'ayant pas été non plus réédité depuis, nous devons attendre l'année 2000 pour avoir accès à l'édition de La danse des vivants du professeur Alain Riffaud, qui rassemble les 160 estampes de la série ordonnées et organisées comme Jean Bruller avait prévu et qui propose aussi une notice avec de précieuses informations inédites sur l'ouvrage. Il a de même réédité en 2002 Visions intimes et rassurantes de la guerre. 
Roy, C. (1974). Esprit de la caricature. Dans C. Roy, R. Searle et B. Bornemann (éds.). La Caricature. Art et manifeste du XVI siècle à nos jours (p. 9-24). Genève : Skira.

Tillier, B. (2005). À la charge! La caricature en France de 1789 à 2000. Paris : Les Éditions de l'Amateur.

Tillier, B. (2015). Une grammaire de la caricature. Dans P. Ory, C. Delporte, B. Tillier, L. Bihl, E. Pierrat et al. (éds.). La caricature... et si c'était sérieux? Décryptage de la violence satirique (p. 31-39). Paris : Nouveau Monde.

Vaillant, A. (2014). Esthétique du rire. Nanterre : Presses universitaires de Paris Nanterre. http://books.openedition.org/pupo/2303

Van der Linden, S. (2008). L'album, le texte et l'image. Le français aujourd'hui, 161, 51-58.

Vercors (1982). Les occasions perdues. L'après Briand (1932-1942). Paris : Plon.

Vercors (1991). À dire vrai. Entretiens de Vercors avec Gilles Plazy. Paris : F. Bourin.

Vercors (2002). La bataille du silence. A. Riffaud (éd.). Paris : Omnibus.

\section{FIGURES}

Bruller, J. (2000). Dimanche ! Dimanche! Dans La danse des vivants. A. Riffaud (éd.). Le Mans : Création \& recherche, estampe 99.

Bruller, J. (2000). Fin de journée ou la vie oisive. Dans La danse des vivants. A. Riffaud (éd.). Le Mans : Création \& recherche, estampe 103.

Bruller, J. (2000). Capitulation ou le libre arbitre. Dans La danse des vivants. A. Riffaud (éd.). Le Mans : Création \& recherche, estampe 108.

Bruller, J. (2000). Symphonie pastorale ou le bienheureux aveugle et sourd. Dans La danse des vivants. A. Riffaud (éd.). Le Mans : Création \& recherche, estampe 148.

Bruller, J. (2000). Le Marchand de canons. Dans La danse des vivants. A. Riffaud (éd.). Le Mans : Création \& recherche, estampe 156.

Bruller, J. (2000). Le Mouchard. Dans La danse des vivants. A. Riffaud (éd.). Le Mans : Création \& recherche, estampe 157.

Bruller, J. (2000). Le Multimilliardaire. Dans La danse des vivants. A. Riffaud (éd.). Le Mans : Création \& recherche, estampe 158.

Bruller, J. (2002). Vision intime de la guerre de Robert Lefrançois industriel. Dans Visions intimes et rassurantes de la guerre. A. Riffaud (éd.). Paris : Omnibus, p. 81.

Bruller, J. (2002). Vision intime de la guerre de Marceline Merlet, gérante des « Mille Dentelles». Dans Visions intimes et rassurantes de la guerre. A. Riffaud (éd.). Paris : Omnibus, p. 61.

Bruller, J. (2002). Vision intime de la guerre de Manuel Trécult, dit Manolo-Les-BellesFeuilles. Dans Visions intimes et rassurantes de la guerre. A. Riffaud (éd.). Paris : Omnibus, p. 63.

Bruller, J. (2002). Vision intime de la guerre du lieutenant-colonel Dupin, officier breveté. Dans Visions intimes et rassurantes de la guerre. A. Riffaud (éd.). Paris : Omnibus, p. 75. 
RÉSUMÉ : Les premiers albums du dessinateur Jean Bruller proposent une caricature sociale originale par la combinaison du texte et de l'image. Si La danse des vivants (1932-1938) aspire à illustrer la comédie humaine du XX $\mathrm{XX}^{\mathrm{e}}$ siècle par la caricature de mœurs et un ton universaliste, Visions intimes et rassurantes de la guerre (1936) s'ancre dans la période de l'entre-deux-guerres pour développer une critique sociale fortement influencée par les menaces de guerre. Cet article propose une analyse approfondie de l'esthétique de ces deux ouvrages brulleriens, marqués par un humour particulièrement mordant et ironique et par un certain arrière-goût pessimiste. Cette approche analytique se nourrira de même du dialogue entre l'œuvre et la pensée de l'artiste, ainsi que du dialogue entre sa production et le contexte historico-social.

Mots-clés : Jean Bruller, Vercors, dessin, album, caricature, $\mathrm{XX}^{\mathrm{e}}$, homme

\section{Social caricature in Jean Bruller's albums: a human comedy of the interwar years}

ABSTRACT: The first albums of the cartoonist Jean Bruller propose an original social caricature by combining text and image. La danse des vivants (1932-1938) aspires to illustrate the human comedy of the 20th century with a caricature of customs and a universalist tone; Visions intimes et rassurantes de la guerre (1936) is anchored in the interwar years to develop a social criticism strongly influenced by the threats of war. This article offers an in-depth analysis of the aesthetics of Jean Bruller's two works, marked by a particularly wry and ironic humour and some pessimism. This analytical approach will also be complemented by an account of the dialogue between the work and the artist's thinking, as well as of the dialogue between his production and the historical-social context.

Keywords: Jean Bruller, Vercors, drawing, album, caricature, 20th century, mankind 